

Л. Г. Афанасьева, Е. Е. Баштова, Вероятности больших уклонений для системы обслуживания с регенерирующим входящим потоком, Теория вероятн. и ее примен., 2015, том 60, выпуск 1, 171-177

DOI: https://doi.org/10.4213/tvp4612

Использование Общероссийского математического портала Math-Net.Ru подразумевает, что вы прочитали и согласны с пользовательским соглашением http: //www . mathnet.ru/rus/agreement

Параметры загрузки:

IP : 3.82 .47 .9

26 апреля 2023 г., 13:40:18




4. Mandelbrot B. B., Van Ness J. W. Fractional Brownian motions, fraxtional noises and applications. - SIAM Rev., 1968, v. 10, № 4, p. 422-437.

5. Peskir G., Shiryaev A. Optimal Stopping and Free-Boundary Problems. ETH Zürich: Springer, 2006, $502 \mathrm{p}$.

6. Norros I., Valkeila E., Virtamo J. An elementary approach to a Girsanov formula and other analytical results on fractional Brownian motions. - Bernoulli, 1999, v. 5, № 4, p. $571-587$.

7. Колмогоров A. Н. Спираль Винера и некоторые другие интересные кривые в гильбертовом пространстве. - Докл. АН СССР, 1940, т. 26, с. 115-118.

8. Липчер P. ШШ., Ширяев А. Н. Статистика случайных процессов: Нелинейная фильтрация и смежные вопросы. М.: Наука, 1974, 696 с.

Поступила в редакцию

12.II.2015

(C) 2015 г. АФАНАСЬЕВА Л. Г. ${ }^{*}$, БАШТОВА Е. Е.

\title{
ВЕРОЯТНОСТИ БОЛЬШИХ УКЛОНЕНИЙ ДЛЯ СИСТЕМЫ ОБСЛУЖИВАНИЯ С РЕГЕНЕРИРУЮЩИМ ВХОДЯЩИМ ПОТОКОМ ${ }^{1)}$
}

\begin{abstract}
Рассматривается одноканальная система обслуживания с неограниченной очередью и регенерирующим входящим потоком. Получены условия, при которых существует ненулевой логарифмический предел для вероятностей больших уклонений времени ожидания в стационарном режиме. Показано, что асимптотическое поведение большой очереди в стационарном режиме определяется совместным распределением периода регенерации входящего потока и числа требований, поступивших за этот период, и не зависит от распределения моментов поступления требований на периоде регенерации.
\end{abstract}

Ключевые слова и фразы: большие уклонения, регенерирующий поток, время ожидания.

1. Введение. Вопросам больших уклонений для распределений функционалов, заданных на траекториях случайных процессов, в частности случайных блужданий, цепей Маркова и процессов восстановления, посвящена обширная литература (см., например, [2], [7], [8], [10], [11] и библиографию в этих работах). Как отмечено в [8], для теории очередей эти вопросы важны не только в теоретическом плане, но и с прикладной точки зрения. Оценка вероятности неприемлемо большой очереди, например, в системе $G|G| r \mid \infty$ позволяет обосновать необходимость принятия тех или иных организационных решений (таких как добавление прибора, увеличение скорости обслуживания, установка буфера конечного объема и т.п.).

Мы рассматриваем одноканальную систему обслуживания с регенерирующим входящим потоком, являющимся обобщением многих процессов, обычно используемых в теории массового обслуживания для описания входящих потоков. Основная цель исследования - определение условий, при которых существует ненулевой логарифмический предел для вероятностей больших уклонений виртуального времени ожидания $W(t)$ в стационарном режиме. Рассматриваются также время ожидания

* Московский государственный университет им. М.В. Ломоносова, механикоматематический факультет, кафедра теории вероятностей, Москва, Россия; e-mail: afanas@mech.math.msu.su; bashtovaelena@rambler.ru

1) Работа выполнена при поддержке РФФИ (грант № 13-01-00653). 
$n$-го требования $w_{n}$ и суммарное остаточное время обслуживания $W_{n}$ требований, находящихся в системе в момент начала $n$-го периода регенерации входящего потока. В предположении, что выполнено условие эргодичности [4], доказывается предельная теорема о больших уклонениях этих процессов. Наш анализ является обобщением хорошо известной характеризации большой очереди в системе $G I|G I| 1 \mid \infty$, полученной с помощью экспоненциального изменения меры и результатов теории восстановления $([3$, гл. ХІІ $],[1$, гл. 4]).

При исследовании больших уклонений для стационарного распределения процесса $w_{n}$ мы опираемся на тот факт, что последовательность интервалов между поступлениями требований регенерирующего потока слабо сходится к стационарной [4]. Это позволяет использовать теорему для систем со стационарной управляющей последовательностью из [8]. Проверка ее условий основана на асимптотике решения уравнения восстановления с несобственной функцией распределения [3, гл. ХI $]$.

Оказалось, что асимптотическое поведение большой очереди в стационарном режиме определяется совместным распределением периода регенерации $\tau_{j}$ и числа требований $\xi_{j}$, поступивших на нем. Это означает, что в области больших уклонений скорость роста очереди с регенерирующим входящим потоком такая же, как если бы через интервалы $\tau_{j}$ поступали группы требований объема $\xi_{j}$, и неважно, как распределены моменты поступления требований в течение периода регенерации, несмотря на то что для регенерирующего потока случайные величины $\xi_{j}$ и $\tau_{j}$, вообще говоря, зависимы. Заметим также, что еще меньше информации необходимо для асимптотического анализа очереди в условиях высокой загрузки. Например, как следует из [4], при $\rho \uparrow 1$, где $\rho$ - коэффициент загрузки, имеет место $C$-сходимость нормированного процесса длины очереди к диффузионному, параметры которого определяются через $\mathbf{D} \xi_{j}, \mathbf{D} \tau_{j}, \operatorname{cov}\left(\xi_{j}, \tau_{j}\right)$.

2. Описание модели и основные обозначения. Рассматривается одноканальная система обслуживания с неограниченной очередью и регенерирующим входящим потоком $X(t)$, определенным на вероятностном пространстве $(\Omega, \mathscr{F}, \mathbf{P})$ с фильтрацией $\left\{F_{t}, t \geqslant 0\right\}$, к которой $X(t)$ адаптирован.

О п р е д е л е н и е 1 . Случайный процесс $(X(t), t \geqslant 0)$ с неубывающими непрерывными слева траекториями назовем регенерирующим потоком, если существует неубывающая последовательность $\left\{\theta_{j}\right\}_{j=1}^{\infty}\left(\theta_{0}=0\right)$ марковских моментов относительно фильтрации $\left\{F_{t}, t \geqslant 0\right\}$ такая, что последовательность

$$
\left\{\kappa_{j}\right\}_{j=1}^{\infty}=\left\{\theta_{j}-\theta_{j-1}, X\left(\theta_{j-1}+t\right)-X\left(\theta_{j-1}\right), t \in\left[0, \theta_{j}-\theta_{j-1}\right)\right\}_{j=1}^{\infty}
$$

состоит из независимых одинаково распределенных случайных элементов.

Случайные величины $\theta_{j}$ и $\tau_{j}=\theta_{j}-\theta_{j-1}(j=1,2, \ldots)$ называются соответственно моментами и периодами регенерации. В теории массового обслуживания $X(t)$ представляет собой либо суммарное время обслуживания требований, либо число требований, поступивших в систему за время $[0, t]$.

Здесь мы считаем, что $X(t)$ - число поступивших требований, и обозначаем $\xi_{j}=$ $X\left(\theta_{j}\right)-X\left(\theta_{j-1}+0\right)$. Далее предполагается, что $a=\mathbf{E} \xi_{1}<\infty$ и $\mu=\mathbf{E} \tau_{1}<\infty$. Тогда с вероятностью 1 существует предел $\lambda=\lim _{t \rightarrow \infty} X(t) / t=a / \mu$, который называется интенсивностью потока $X(t)$.

Заметим, что класс регенерирующих потоков весьма широк и включает значительную часть потоков, обычно используемых в теории массового обслуживания в качестве входящих. Например, дважды стохастический пуассоновский процесс [9] с интенсивностью, являющейся регенерирующим процессом, будет регенерирующим потоком. K классу регенерируюших потоков также относятся полумарковский [5], марковски модулированный, марковский потоки поступлений [6] и многие другие. Кроме того, регенерирующий поток обладает рядом свойств; некоторые из них приведены и доказаны в [4]. Свойства регенерирующего потока позволяют использовать 
подходы, разработанные для классических моделей обслуживания. Именно это будет продемонстрировано в настоящей статье.

Далее предполагается выполненным следующее условие.

Условие 1. Решетчатый процесс восстановления, соответствующий последовательности $\left\{\xi_{j}\right\}_{j=1}^{\infty}$, апериодичен, т.е.

$$
\text { н.о.д. }\left\{k: \mathbf{P}\left(\xi_{1}=k\right)>0\right\}=1 \text {. }
$$

Нам понадобится следующее свойство регенерирующего потока, доказанное в [4]. Пусть $t_{n}-$ момент поступления $n$-го требования, а $\zeta_{n}=t_{n}-t_{n-1}\left(t_{0}=0\right)$. Тогда при выполнении условия 1 последовательность $\left\{\zeta_{n+k}\right\}_{k=1}^{\infty}$ при $n \rightarrow \infty$ слабо сходится к стационарной.

Заметим, что распределение стохастического процесса $X(t)$ определяется распределением случайного элемента $\kappa_{1}$. Однако для асимптотического анализа вероятностей больших уклонений очереди в системе обслуживания с регенерирующим входящим потоком это распределение не потребовалось. Оказалось достаточным знание совместного распределения $\xi_{1}$ и $\tau_{1}$, т.е. функции

$$
G(z, s)=\mathbf{E} e^{-s \tau_{1}} z^{\xi_{1}} \quad(\operatorname{Re} s \geqslant 0,|z|<1) .
$$

Итак, мы рассматриваем одноканальную систему обслуживания с регенерирующим входящим потоком $X(t)$ и неограниченным числом мест для ожидания, которую, следуя символике Кендалла, можно обозначить $\operatorname{Reg}|G I| 1 \mid \infty$.

Времена обслуживания требований $\left\{\eta_{i}\right\}_{i=1}^{\infty}$ образуют последовательность независимых случайных величин с общей функцией распределения $B(x)$, средним $b<\infty$ и преобразованием Лапласа-Стилтьеса $b(s)=\int_{0}^{\infty} e^{-s x} d B(x)(\operatorname{Re} s \geqslant 0)$. Введем процесс виртуального времени ожидания $W(t)$, а также вложенные процессы

$$
w_{n}=W\left(t_{n}-0\right), \quad W_{n}=W\left(\theta_{n}-0\right) .
$$

Как показано в [4], у всех этих процессов собственное предельное распределение существует тогда и только тогда, когда коэффициент загрузки $\rho=\lambda b$ меньше 1 . Далее это условие предполагается выполненным. Положим

$$
\Phi(x)=\lim _{n \rightarrow \infty} \mathbf{P}\left(W_{n} \leqslant x\right), \quad F(x)=\lim _{n \rightarrow \infty} \mathbf{P}\left(w_{n} \leqslant x\right) .
$$

Наш первый результат касается поведения функций $1-\Phi(x)$ и $1-F(x)$ при $x \rightarrow \infty$. А именно, при определенных условиях будет доказано существование логарифмических пределов для этих стационарных распределений при $x \rightarrow \infty$.

\section{3. Основной результат.}

Теорема 1. Пусть выполнено условие 1 и

$$
\begin{gathered}
\delta_{0}=\sup \{s: G(b(-s), s)<\infty\}>0, \\
G\left(b\left(-\delta_{0}\right), \delta_{0}\right)>1 .
\end{gathered}
$$

Тогда существуют предель

$$
\begin{aligned}
& \lim _{x \rightarrow \infty} \frac{1}{x} \ln (1-F(x))=-q, \\
& \lim _{x \rightarrow \infty} \frac{1}{x} \ln (1-\Phi(x))=-q,
\end{aligned}
$$

где $q$ - единственное положительное решение уравнения

$$
G(b(-s), s)=1 .
$$


Д о к а з а т е л ь с т в о. Начнем с доказательства соотношения (4). В силу слабой сходимости последовательности $\left\{\zeta_{n+k}\right\}_{k=1}^{\infty}$ при $n \rightarrow \infty$ к стационарной можно считать, не ограничивая общности, что $\left\{\zeta_{n}\right\}_{n=1}^{\infty}-$ стационарная последовательность.

Здесь мы будем исходить из результатов главы 3 в [8], которые применительно к нашей ситуации могут быть сформулированы следующим образом. Пусть $\left\{u_{j}\right\}_{j=1}^{\infty}-$ стационарная последовательность случайных величин с отрицательным математическим ожиданием и $U_{n}=\sum_{j=1}^{n} u_{j}, U_{0}=0$. Обозначим

$$
\Lambda_{n}(s)=\ln \mathbf{E} e^{s U_{n}}
$$

и предположим, что существует предел

$$
\lim _{n \rightarrow \infty} \frac{1}{n} \Lambda_{n}(s)=\Lambda(s) .
$$

Тогда, если в некоторой окрестности нуля функция $\Lambda_{n}(s)$ конечна при всех $n$, а функция $\Lambda(s)$ дифференцируема, то существует логарифмический предел

$$
\lim _{x \rightarrow \infty} \frac{1}{x} \ln \mathbf{P}\left(\sup _{n \geqslant 0} U_{n}>x\right)=-q
$$

где $q=\sup \{s>0: \Lambda(s)<0\}$.

Как хорошо известно (см., например, [1]),

$$
F(x)=\mathbf{P}\left(\sup _{n \geqslant 0} U_{n}<x\right)
$$

где $u_{j}=\eta_{j}-\zeta_{j}, j=1,2, \ldots$, так что для применения сформулированного результата из [8] нам необходимо установить (7) для функции

$$
\Lambda_{n}(s)=\ln \mathbf{E} \exp \left\{s \sum_{j=1}^{n}\left(\eta_{j}-\zeta_{j}\right)\right\} .
$$

Лемма 1. В условиях теоремь 1 при $s \geqslant 0$ существует предел

$$
\lim _{n \rightarrow \infty} \frac{1}{n} \Lambda_{n}(s)=\ln \frac{b(-s)}{z_{1}(s)},
$$

где $\Lambda_{n}(s)$ определено в (8), а $z_{1}(s)-$ единственное решение уравнения

$$
G(z, s)=1
$$

относительно z такое, ито $z_{1}(s)>1$ при $s>0$.

Д о к а з а т е л ь с т в о. В силу независимости последовательностей $\left\{\eta_{j}\right\}_{j=1}^{\infty}$ и $\left\{\zeta_{j}\right\}_{j=1}^{\infty}$ имеем

$$
\Lambda_{n}(s)=\ln \mathbf{E} \exp \left\{s \sum_{j=1}^{n} \eta_{j}\right\}+\ln \mathbf{E} e^{-s t_{n}}=n \ln b(-s)+\ln \mathbf{E} e^{-s t_{n}} .
$$

Поэтому нам достаточно доказать, что существует предел

$$
\lim _{n \rightarrow \infty} \frac{1}{n} \ln \mathbf{E} e^{-s t_{n}}=\ln \frac{1}{z_{1}(s)} .
$$

Обозначим $\nu(n)=\min \left\{k: \xi_{1}+\cdots+\xi_{k} \geqslant n\right\}$, так что $\nu(n)-$ номер периода регенерации, на котором поступило $n$-е требование. Тогда

$$
\theta_{\nu(n)-1} \leqslant t_{n}<\theta_{\nu(n)} \quad\left(\theta_{0}=0\right) .
$$

Сначала установим, что (11) выполнено для $\theta_{\nu(n)}$, т.е.

$$
\lim _{n \rightarrow \infty} \frac{1}{n} \ln \mathbf{E} e^{-s \theta_{\nu(n)}}=\ln \frac{1}{z_{1}(s)} .
$$


При $n \geqslant 1$ имеем стохастическое равенство

$$
\theta_{\nu(n)}=\sum_{j=0}^{n-1}\left(\tau_{1}+\widetilde{\theta}_{\nu(n-j)}\right) \chi\left(\xi_{1}=j\right)+\tau_{1} \chi\left(\xi_{1} \geqslant n\right),
$$

где $\chi(A)$ - индикаторная функция события $A$, случайная величина $\widetilde{\theta}_{\nu(n-j)}$ не зависит от $\tau_{1}$ и $\xi_{1}$ и по распределению совпадает с моментом окончания периода регенерации, на котором поступило $(n-j)$-е требование. Положим

$$
\begin{aligned}
\varphi_{n}(s) & =\mathbf{E} e^{-s \theta_{\nu}(n)}, \\
g_{j}(s) & =\mathbf{E}\left(e^{-s \tau_{1}} \chi\left(\xi_{1}=j\right)\right), \\
\psi_{n}(s) & =\mathbf{E}\left(e^{-s \tau_{1}} \chi\left(\xi_{1} \geqslant n\right)\right) .
\end{aligned}
$$

Из (14) находим, что

$$
\varphi_{n}(s)=\psi_{n}(s)+\sum_{j=0}^{n-1} \varphi_{n-j}(s) g_{j}(s) .
$$

Зафиксируем $s>0$. Тогда (15) представляет собой уравнение восстановления, соответствующее обрывающемуся процессу восстановления $\left\{\widetilde{\xi}_{n}\right\}_{n=1}^{\infty}$, где $\mathbf{P}\left(\widetilde{\xi}_{n}=j\right)=g_{j}(s)$ и $\mathbf{P}\left(\widetilde{\xi}_{n}=\infty\right)=1-\mathbf{E} e^{-s \tau_{1}}$.

Поскольку функция $G(z, s)$ при каждом $s>0$ строго монотонно возрастает по $z$ и $G(1, s)<1$, а $G(\infty, s)=\infty$, то существует единственное решение $z_{1}(s)>1$ уравнения (10).

Так как $\psi_{n}(s) \rightarrow 0(n \rightarrow \infty)$, а $\varphi_{n}(s)$ удовлетворяет (15), то на основе теоремы 2 из [3, гл. XI, §6] получаем, что при $n \rightarrow \infty$

$$
\varphi_{n}(s) \sim \frac{C}{\left(z_{1}(s)\right)^{n}}
$$

где $C$ - некоторая константа. Как обычно, мы пишем $a_{n} \sim C b_{n}$, если $\lim _{n \rightarrow \infty} a_{n} / b_{n}=$ $C$. Соотношение (16) означает выполнение (13). Аналогичным образом (13) устанавливается для $\theta_{\nu(n)-1}$, откуда ввиду (12) следует (11).

Таким образом, имеем

$$
\Lambda(s)=\lim _{n \rightarrow \infty} \frac{1}{n} \Lambda_{n}(s)=\ln \frac{b(-s)}{z_{1}(s)} .
$$

В силу (2) и (3) функция $\Lambda_{n}(s)$ конечна при $s<\delta_{0}$ для каждого $n \geqslant 1$, а $\Lambda(s)$ удовлетворяет условиям теоремы 3.1 из [8], так что имеет место (4). При этом $q-$ единственный положительный корень уравнения

$$
b(-s)=z_{1}(s) .
$$

Поскольку $z_{1}(s)$ удовлетворяет уравнению (10), то $q$ является единственным положительным корнем уравнения (6). Действительно, рассмотрим функцию $f(s)=$ $G(b(-s), s)$. Имеем $f(0)=1, f^{\prime}(0)=b a-\mu<0$ в силу условия эргодичности и $f\left(\delta_{0}\right)>1$ в силу (3). Это означает, что уравнения (6) и (17) имеют единственный положительный корень $q$.

Перейдем к доказательству соотношения (5). Пусть $\gamma_{n}$ - суммарное время обслуживания требований, поступивших в систему на $n$-м периоде регенерации. Введем вспомогательный процесс $W_{n}^{-}$с помощью рекуррентных соотношений

$$
W_{n}^{-}=\left[W_{n-1}^{-}+\gamma_{n}-\tau_{n}\right]^{+}, \quad W_{0}^{-}=0 .
$$

Очевидно, что при $W_{0}=0$ с вероятностью 1 справедливо неравенство

$$
W_{n} \geqslant W_{n}^{-} .
$$


Обозначим $\Phi^{-}(x)=\lim _{n \rightarrow \infty} \mathbf{P}\left(W_{n}^{-} \leqslant x\right)$. Поскольку $\mathbf{E} e^{s\left(\gamma_{n}-\tau_{n}\right)}=G(b(-s), s)$, то в силу (2) и (3) при $x \rightarrow \infty$

$$
1-\Phi^{-}(x)=\mathbf{P}\left(\sup _{n \geqslant 0} S_{n}>x\right) \sim C e^{-q x},
$$

где, как и раньше, $q$ - единственное положительное решение уравнения $(6), S_{n}=$ $\sum_{j=1}^{n}\left(\gamma_{j}-\tau_{j}\right), S_{0}=0$ и $C-$ некоторая константа (см., например, [1], [3]).

Отсюда и из (18) следует, что

$$
\liminf _{x \rightarrow \infty} \frac{1}{x} \ln (1-\Phi(x)) \geqslant-q .
$$

Получим оценку для верхнего предела. Пусть $\mu(n)=\xi_{1}+\cdots+\xi_{n}-$ число требований, поступивших за первые $n$ периодов регенерации, так что $\mu(n)-$ номер требования, поступившего последним перед моментом $\theta_{n}$. Если $\mu(n)=0$, полагаем $w_{0}=0$, $\eta_{0}=0 \mathrm{c}$ вероятностью 1 . Тогда

$$
W_{n} \leqslant w_{\mu(n)}+\eta_{\mu(n)}
$$

и величины $w_{\mu(n)}$ и $\eta_{\mu(n)}$ независимы. Из усиленного закона больших чисел и определения функции $F(x)$ следует, что $\lim _{n \rightarrow \infty} \mathbf{P}\left(w_{\mu(n)} \leqslant x\right)=F(x)$, и поэтому

$$
\lim _{n \rightarrow \infty} \mathbf{P}\left(W_{n}>x\right) \leqslant \int_{0}^{x}(1-F(x-y)) d B(y)+1-B(x) .
$$

В силу (4) для любого $\varepsilon>0$ существует $x_{\varepsilon}$ такое, что при $x \geqslant x_{\varepsilon}$ выполнено неравенство $1-F(x) \leqslant e^{-(q-\varepsilon) x}$. Значит, для некоторого $C_{\varepsilon}$ при всех $x \geqslant 0$ будет выполняться неравенство

$$
1-F(x) \leqslant C_{\varepsilon} e^{-(q-\varepsilon) x} .
$$

Обозначим $\delta_{1}=\sup \{s: b(-s)<\infty\}$. Заметим, что если $s<\delta_{0}$, то $G(b(-s), s)<\infty$, а следовательно, $b(-s)<\infty$. Это означает, что $\delta_{1} \geqslant \delta_{0}$, и, так как $q<\delta_{0}$, имеем $b(-q)<\infty$. Значит, найдутся $\delta_{2}>q$ и $C_{1}>0$ такие, что $1-B(x) \leqslant C_{1} e^{-\delta_{2} x}$. Из (21) и (22) при $x \rightarrow \infty$ получаем

$$
\begin{aligned}
1-\Phi(x) & \leqslant C_{\varepsilon} e^{-(q-\varepsilon) x} \int_{0}^{\infty} e^{q y} d B(y)+C_{1} e^{-\delta_{2} x} \\
& \leqslant C_{\varepsilon} e^{-(q-\varepsilon) x} b(-q)(1+o(1))
\end{aligned}
$$

так что

$$
\limsup _{x \rightarrow \infty} \frac{1}{x} \ln (1-\Phi(x)) \leqslant-q+\varepsilon .
$$

Поскольку $\varepsilon$ сколь угодно мало, это вместе с (19) доказывает (5).

4. Асимптотика распределения виртуального времени ожидания. Здесь мы рассмотрим функцию

$$
\Psi(x)=\lim _{t \rightarrow \infty} \mathbf{P}(W(t) \leqslant x),
$$

предположив, как и раньше, что $\rho=\lambda b<1$.

Следствие 1. В условиях теоремы 1 существует предел

$$
\lim _{x \rightarrow \infty} \frac{1}{x} \ln (1-\Psi(x))=-q,
$$

где $q$ - единственное положительное решение уравнения (6).

Д о к а з а т е л ь с т в о. Обозначим $l(t)$ номер периода регенерации входящего потока, на который попадает момент $t$, а $n(t)$ номер требования, пришедшего последним в интервале $[0, t)$. Тогда при $x \geqslant 0$ выполнены неравенства

$$
\begin{aligned}
\mathbf{P}\left(W_{l(t)-1}-\tau_{l(t)}>x\right) & \leqslant \mathbf{P}(W(t)>x) \\
& \leqslant \mathbf{P}\left(w_{n(t)}+\eta_{n(t)}>x\right) .
\end{aligned}
$$


При этом величины $W_{l(t)}$ и $\tau_{l(t)}$, а также $w_{n(t)}$ и $\eta_{n(t)}$ независимы. Кроме того, из усиленного закона больших чисел следует, что

$$
\begin{aligned}
& \lim _{t \rightarrow \infty} \mathbf{P}\left(W_{l(t)} \leqslant x\right)=\Phi(x), \\
& \lim _{t \rightarrow \infty} \mathbf{P}\left(w_{n(t)} \leqslant x\right)=F(x),
\end{aligned}
$$

а из основной теоремы восстановления вытекает существование предела

$$
\lim _{t \rightarrow \infty} \mathbf{P}\left(\tau_{l(t)} \leqslant x\right)=\frac{1}{\mu}\left(\int_{0}^{x} \mathbf{P}\left(\tau_{1}>y\right) d y-x \mathbf{P}\left(\tau_{1}>x\right)\right) .
$$

Переходя в (24) к пределу при $t \rightarrow \infty$, находим

$$
\begin{aligned}
\frac{1}{\mu} \int_{0}^{\infty} y(1-\Phi(x+y)) d \mathbf{P}\left(\tau_{1} \leqslant y\right) & \leqslant 1-\Psi(x) \\
& \leqslant \int_{0}^{x}(1-F(x-y)) d B(y)+1-B(x) .
\end{aligned}
$$

Поскольку для $1-F(x)$ и $1-\Phi(x)$ существуют логарифмические пределы $(4)$ и $(5)$ соответственно, а $1-B(x)=o\left(e^{-(q-\varepsilon) x}\right)$ при $x \rightarrow \infty$, то при достаточно малом $\varepsilon>0$ имеем

$$
\begin{aligned}
& \liminf _{x \rightarrow \infty} \frac{1}{x} \ln (1-\Psi(x)) \geqslant-q-\varepsilon, \\
& \limsup _{x \rightarrow \infty} \frac{1}{x} \ln (1-\Psi(x)) \leqslant-q+\varepsilon .
\end{aligned}
$$

Это, ввиду произвольной малости $\varepsilon$, доказывает (23).

\section{СПИСОК ЛИТЕРАТУРЫ}

1. Боровков A. A. Вероятностные процессы в теории массового обслуживания. М.: Наука, 1972, 367 с.

2. Боровков A.A., Боровков K.A. Вероятности больших уклонений для обобщенных процесссов восстановления с правильно меняющимися распределениями скачков. - Матем. тр., 2005, т. 8, № 2, с. 69-136.

3. Феллер B. Введение в теорию вероятностей и ее приложения, т. 2. М.: Мир, 1984, $752 \mathrm{c}$.

4. Afanasyeva L., Bashtova E. Coupling method for asymptotic analysis of queues with regenerative input and unreliable server. - Queueing Syst., 2014, v. 76, № 2, p. 125147.

5. Afanasyeva L., Bashtova E., Bulinskaya E. Limit theorems for semi-Markov queues and their applications. - Comm. Statist. Simulation Comput., 2012, v. 41, № 6, p. 688-709.

6. Asmussen S. Applied Probability and Queues. Chichester: Wiley, 1987, 318 p.

7. Bryc W., Dembo A. Large deviations and strong mixing. - Ann. Inst. H. Poincaré, 1996, v. 32, № 4, p. 549-569.

8. Ganesh A., O'Connell N., Wischik D. Big queues. - Lecture Notes in Math., 2004, v. 1838 , p. $1-254$.

9. Grandell J. Double stochastic Poisson processes. - Lecture Notes in Math., 1976, v. 529, p. $1-234$.

10. Sadowsky J.S., Szpankowski $W$. The probability of large queue lengths and waiting times in a heterogeneous multiserver queue. I. Tight limits. - Adv. Appl. Probab., 1995 , v. 27 , № 2 , p. 532-566.

11. Sadowsky J. S. The probability of large queue length and waiting times in a heterogeneous multiserver queue. II. Positive recurrence and logarithmic limits. - Adv. Appl. Probab., 1995, v. 27, № 2, p. 567-583. 